\title{
Tetraquarks composed of 4 heavy quarks
}

\author{
A. V. Luchinsky* \\ Institute for High Energy Physics, Protvino, 142281 Russian Federation \\ E-mail: Alexey.Luchinsky@ihep.ru
}

In the current work spectroscopy and possibility of observation at the LHC of tetraquarks composed of 4 heavy quarks is discussed. Tetraquarks concerned are $T_{4 c}=[c c][\bar{c} \bar{c}], T_{4 b}=[b b][\bar{b} \bar{b}]$ and $T_{2[b c]}=[b c][\bar{b} \bar{c}]$. By solving nonrelativistic Schroedinger equation masses of these states are found with the hyperfine splitting accounted for. It is shown that masses of tensor tetraquarks $T_{4 c}\left(2^{++}\right)$and $T_{2[b c]}\left(2^{++}\right)$are high enough to observe these states as peaks in the invariant mass distributions of heavy quarkonia pairs in $p p \rightarrow T_{4 c}+X \rightarrow 2 J / \psi+X, p p \rightarrow T_{2[b c]}+X \rightarrow 2 B_{c}+X$ and $p p \rightarrow T_{2[b c]}+X \rightarrow J / \psi \Upsilon(1 S)+X$ channels while $T_{4 b}$ is under the threshold of decay into a vector bottomonia pair.

LHC on the March,

November 16-18, 2011

Protvino, Moscow region, Russian Federation

${ }^{*}$ Speaker. 


\section{Introduction}

Recent observation of $J / \psi$-meson pairs production in proton-proton collisions at $7 \mathrm{TeV}$ energy at LHC renewed interest to the 4 heavy quarks final states. In the low invariant mass region these quarks can form bound states (called tetraquarks) which can be produced in hadronic experiments. Thereby we would like to discuss physics of these states, elaborate their mass spectrum and possibility of experimental observation.

Conception of tetraquarks, i.e. mesons composed of 4 valent quarks $(q q \bar{q} \bar{q})$, was first introduced in works $[1,2]$ in 1976. For example, $a_{0}$-meson and $\sigma$-mesons was treated as possible tetraquark candidate [3-6]. However, it is hard to determine quark composition of a particle in the light meson domain so these ideas were not developed further. Observation of new unexpected states such as $X(3872)[7,8]$ gave this idea a new impetus [9, 10]. Eccentricity of these particles consists in fact that according to the modes of their production and decay they contain a $c \bar{c}$ pair but they can not be included in the well known systematics of charmonia. Later similar particles were also found in the bottomonia sector [11-14]. It is natural to ascribe these mesons to tetraquarks $(Q q \bar{Q} \bar{q})$, where $q$ are light and $Q$ - heavy ( $b$ or $c$ ) quarks.

However, situation when all quarks composing a tetraquark are heavy was not treated in details yet. This possibility seems to be quite interesting as in this case determination of meson's quark composition becomes simpler and its parameters can be determined by solving nonrelativistic Schroedinger equation. Our work is devoted to these particular questions.

In our recent paper [15] we consider tetraquark $T_{4 c}=[c c][\bar{c} \bar{c}]$ in the framework of diquark model.The hyperfine splitting in that paper was described through interaction of total diquark spins. Now we would like to study also tetraquarks $T_{4 b}=[b b][\bar{b} \bar{b}]$ and $T_{2[b c]}=[b c][\bar{b} \bar{c}]$. The last state is especially interesting since, in contrast to tetraquarks build from four identical quarks, both singlet and triplet spin states of the diquark are possible. It is clear, that hyperfine interaction of spinsinglet diquark cannot be described with the method used in our previous paper, so some other approach should be applied.

In the following section spectroscopy of $(c c \bar{c} \bar{c}),(b b \bar{b} \bar{b})$ and $(b c \bar{b} \bar{c})$ tetraquarks is concerned. Possibility of observation of these particles in hadronic experiments is discussed in the third section. We summarize our results in the short conclusion.

\section{Spectroscopy}

In the current study diquark model of tetraquark is used. According to this approach tetraquark

$$
T=Q_{1} Q_{2} \bar{Q}_{3} \bar{Q}_{4}
$$

consists of 2 almost point-like diquarks $\overline{\mathscr{D}}_{12}=\left[Q_{1} Q_{2}\right]$ and $\mathscr{D}_{34}=\left[\bar{Q}_{3} \bar{Q}_{4}\right]$ with certain quantum numbers (such as angular momentum, spin, color) and mass. What concerns color configuration, two quarks in diquark can be in triplet or sextet color state. According to the manuscript [16] in the sextet configuration diquarks experience mutual repulsion so we restrict ourselves to the (anti)triplet color configurations. Angular momentum of the diquark system equals 0 in the ground state, so its spin is equal to the sum of quark spins which is 0 or 1 . What concerns diquark mass, it can be determined by solving Schroedinger equation with a correctly selected potential. According 
to work [17] it is possible to use quark-antiquark interaction potential used in heavy quarkonia calculations with additional factor $1 / 2$ due to the different color structures.

In the diquark model tetraquark mass can be determined by solving 2-particle Schroedinger equation with point-like diquarks. As $\left[Q_{1} Q_{2}\right]$ and $\left[\bar{Q}_{3} \bar{Q}_{4}\right]$ diquarks are in (anti)triplet color configuration, potential of their interaction coincides with that of quark and antiquark in heavy quarkonia. Hyperfine splitting in this system can be described by the hamiltonian [3]

$$
H=M_{0}+2 \sum_{i<j} \kappa_{i j}\left(\mathbf{S}_{i} \mathbf{S}_{j}\right)
$$

where $M_{0}$ is the tetraquark mass without splitting, $\mathbf{S}_{i}$ - spin operator of $i$-th (anti)quark, and $\kappa_{i j}$ are constants determined from experimental data analysis or theoretically. When dealing with potential models, $\kappa_{i j}$ coefficient can be obtained from the value of the $Q_{i} Q_{j}$ system wave function at origin:

$$
\kappa_{i j}=\frac{1}{2} \frac{4}{9 m_{Q_{1}} m_{Q_{2}}} \alpha_{s}\left|R_{[i j]}(0)\right|^{2}
$$

when both $Q_{i}$ and $Q_{j}$ are quarks or antiquarks in the color triplet state, and

$$
\kappa_{i j}=\frac{1}{2} \frac{8}{9 m_{Q_{1}} m_{Q_{2}}} \alpha_{s}\left|R_{(i j)}(0)\right|^{2}
$$

if $Q_{i}$ and $Q_{j}$ are quark and antiquark in color singlet configuration. Three loop expression for the strong coupling constant was used when calculating $\kappa_{i j}$ constants [17] and scale for it was taken equal

$$
\mu^{2}=\frac{2 m_{Q_{1}} m_{Q_{2}}}{m_{Q_{1}}+m_{Q_{2}}}\langle T\rangle
$$

where $\langle T\rangle$ is average kinetic energy of quarks which is equal

$$
\left\langle T_{d}\right\rangle=0.19 \mathrm{GeV}, \quad\left\langle T_{s}\right\rangle=0.38 \mathrm{GeV}
$$

for the triplet and singlet states respectively. Values of diquark wave functions in origin are presented in manuscript [17] while for mesons they can be calculated from the leptonic width $\Gamma_{e e}$ or leptonic constant $f$ of meson in question:

$$
|R(0)|^{2}=\frac{1}{\alpha^{2} e_{q}^{2}} \Gamma_{e e} \frac{M^{2}}{4}=\frac{M^{2} f}{9} .
$$

Up to the hyperfine splitting tetraquark can be described by its total spin $J$, spins of diquarks $S_{12}$ and $S_{34}$ constituting it and its spatial and charge parities $P$ and $C$ :

$$
\begin{aligned}
\left|0^{++}\right\rangle & =|0 ; 0,0\rangle, & \left|0^{++^{\prime}}\right\rangle=|0 ; 1,1\rangle, \\
\left|1^{+ \pm}\right\rangle & =\frac{1}{\sqrt{2}}(|1 ; 0,1\rangle \pm|1 ; 0,1\rangle), & \left|1^{+-^{\prime}}\right\rangle=|1 ; 1,1\rangle, \\
\left|2^{++}\right\rangle & =|2 ; 1,1\rangle &
\end{aligned}
$$


In this treatment all states are degenerate with mass $M_{0}$. If spin-spin interaction is accounted for, masses of $\left|1^{++}\right\rangle$and $\left|2^{++}\right\rangle$states shift:

$$
\begin{aligned}
& M\left(1^{++}\right)=\left\langle 1^{++}|H| 1^{++}\right\rangle=M_{0}-\kappa_{12}-\kappa_{-}, \\
& M\left(2^{++}\right)=M_{0}+\kappa_{12}+\kappa_{+},
\end{aligned}
$$

where following designations are introduced:

$$
\kappa_{ \pm}=\frac{2 \kappa_{14} \pm \kappa_{13} \pm \kappa_{24}}{2},
$$

The states $\left|0^{++}\right\rangle,\left|0^{++^{\prime}}\right\rangle$ and $\left|1^{+-}\right\rangle,\left|1^{+-^{\prime}}\right\rangle$ mix with each other. In the scalar tetraquarks case mixing matrix has the following form:

$$
H\left[\begin{array}{c}
\left|0^{++}\right\rangle \\
\left|0^{++^{\prime}}\right\rangle
\end{array}\right]=\left[\begin{array}{cc}
M_{0}-3 \kappa_{12} & -\sqrt{3} \kappa_{-} \\
-\sqrt{3} \kappa_{-} & M_{0}+\kappa_{12}-2 \kappa_{+}
\end{array}\right]\left[\begin{array}{c}
\left|0^{++}\right\rangle \\
\left|0^{++^{\prime}}\right\rangle
\end{array}\right],
$$

and for $\left|1^{+-}\right\rangle$tetraquarks:

$$
H\left[\begin{array}{c}
\left|1^{+-}\right\rangle \\
\left|1^{+-^{\prime}}\right\rangle
\end{array}\right]=\left[\begin{array}{cc}
M_{0}-\kappa_{12}+\kappa_{-} & \kappa_{13}-\kappa_{24} \\
\kappa_{13}-\kappa_{24} & M_{0}+\kappa_{12}-\kappa_{+}
\end{array}\right]\left[\begin{array}{l}
\left|1^{+-}\right\rangle \\
\left|1^{+-^{\prime}}\right\rangle
\end{array}\right] .
$$

In the case when quarks of the same flavor are involved, Fermi-Dirac statistics leads to the additional restrictions on the diquark quantum numbers. Indeed, permutation of quark indices should change sign of the total diquark wave function. As quarks are in the antitriplet color state, color part of this function is antisymmetric. Radial wave function is symmetric as quarks are in the $S$-wave. So spin part of the wave function should be symmetric too. Consequently the total spin of the $S$-wave diquark can only be equal to 1 . As a result only $\left|0^{++^{\prime}}\right\rangle,\left|1^{+-^{\prime}}\right\rangle$ and $\left|2^{++}\right\rangle$diquark states remain. They do not mix with each other after the spin-spin interaction is accounted for. Masses of these states are equal

$$
\begin{gathered}
M\left(0^{++^{\prime}}\right)=M_{0}+\kappa_{12}-2 \kappa_{+}, \\
M\left(1^{+-^{\prime}}\right)=M_{0}+\kappa_{12}-\kappa_{+}, \\
M\left(2^{++}\right)=M_{0}+\kappa_{12}+\kappa_{+} .
\end{gathered}
$$

It worth mentioning that this splitting scheme agrees with the result of the work [15] in which interaction of the total diquark spins was concerned.

To obtain numerical values of tetraquark masses one needs to know the unsplitted mass $M_{0}$ and coefficients $\kappa_{i j}$ in the Hamiltonian (2.1). These coefficients can be calculated using expressions (2.2) and (2.3). The following values of quark masses were used:

$$
m_{c}=1.468 \mathrm{GeV}, \quad m_{b}=4.873 \mathrm{GeV} .
$$

Diquark masses without hyperfine splitting are given in [17] while $M_{0}$ mass of the tetraquark was calculated using a procedure similar to that described in [17]. Numerical values for these parameters, used in our paper, are presented in table 1. 


\begin{tabular}{|c|c|c|c|c|}
\hline$Q_{1} Q_{2}$ & $\kappa_{\left(Q_{1} \bar{Q}_{2}\right)}, \mathrm{MeV}$ & $\kappa_{\left[Q_{1} Q_{2}\right]}, \mathrm{MeV}$ & $m_{\left[Q_{1} Q_{2}\right]}, \mathrm{GeV}$ & $M_{0}\left(2\left[Q_{1} Q_{2}\right]\right) \mathrm{GeV}$ \\
\hline$c c$ & 42.8 & 12.8 & 3.13 & 6.124 \\
$b b$ & 27.1 & 5.52 & 9.72 & 18.857 \\
$b c$ & 27.1 & 6.43 & 6.45 & 12.491 \\
\hline
\end{tabular}

Table 1: Diquark masses and parameters of spin splitting

Let us begin with the tetraquark composed of $4 c$-quarks, $T_{4 c}=[c c][\bar{c} \bar{c}]$. The masses of tetraquarks of this family are presented in the second coloumn of table 2. One can easily see, that only tensor particle $T_{4 c}\left(2^{++}\right)$lies above the threshold of double $J / \psi$-meson production and cane be observed in the decay $T_{4 c} \rightarrow 2 J / \psi$. As for the family $T_{4 b}$, it turns out that all tetraquarks of this family lie below the threshold of double $\Upsilon(1 S)$ production (see the third column of table 2 ). From the last coloumn of this table it can be easily seen also, that all $T_{2[b c]}$ tetraquarks lie below the theshold of double vector $B_{c}^{*}$ meson production. In contrast to heavy quarkonia with hidden flavour, however, the pseudoscalar meson $B_{c}$ is obseved in the experiment, so it is better to search these tetraquark in the decays $T_{2[b c]} \rightarrow 2 B_{c}$. From table 2 it can be seen, that only tensor meson $T_{2[b c]}\left(2^{++}\right)$lies above the threshold of this reaction.

\begin{tabular}{|c||c|c|c|c|}
\hline$T$ & $T_{4 c}$ & $T_{4 b}$ & \multicolumn{2}{|c|}{$T_{2[b c]}$} \\
\hline$M\left(0^{++}\right)$ & 5.966 & 18.754 & 12.359 & 12.471 \\
\hline$M\left(1^{++}\right)$ & - & - & \multicolumn{2}{|c|}{12.485} \\
\hline$M\left(1^{+-}\right)$ & 6.051 & 18.808 & 12.424 & 12.488 \\
\hline$M\left(2^{++}\right)$ & 6.223 & 18.916 & \multicolumn{2}{|c|}{12.566} \\
\hline
\end{tabular}

Table 2: Tetraquark masses

In paper [18] tetraquark states were also considered in the framework of diquark model. Picture of hyperfine splittings of $T_{2[b c]}$-tetraquark, presented in this paper is in good agreement with our results. Predictions for masses, on the other hand, are about $700 \mathrm{MeV}$ higher, than our values. As a result, according to this paper all tetraqurk states should lie above $2 B_{c}^{*}$ and $J / \psi \Upsilon$ thresholds. We think, that the main reason for difference between these two works is the neglection of binding energy in tetraquark tetraquark and diquark spectra, that is negative. For example, for tetraquark state before hyperfine splitting we have $\delta E=M_{0}-2 m_{[b c]} \approx 410 \mathrm{MeV}$.

\section{Production}

Duality relations can be used to estimate production cross sections of the particles in question. Let us consider formation of two diquarks in gluon interaction $g g \rightarrow\left[Q_{1} Q_{2}\right]\left[\bar{Q}_{3} \bar{Q}_{4}\right]$. Above the 2 doubly-heavy baryon production threshold these diquarks can hadronize into 4 open heavy flavor mesons, 2 heavy quarkonia or form a bound state, i.e. tetraquark. According to our estimations this 


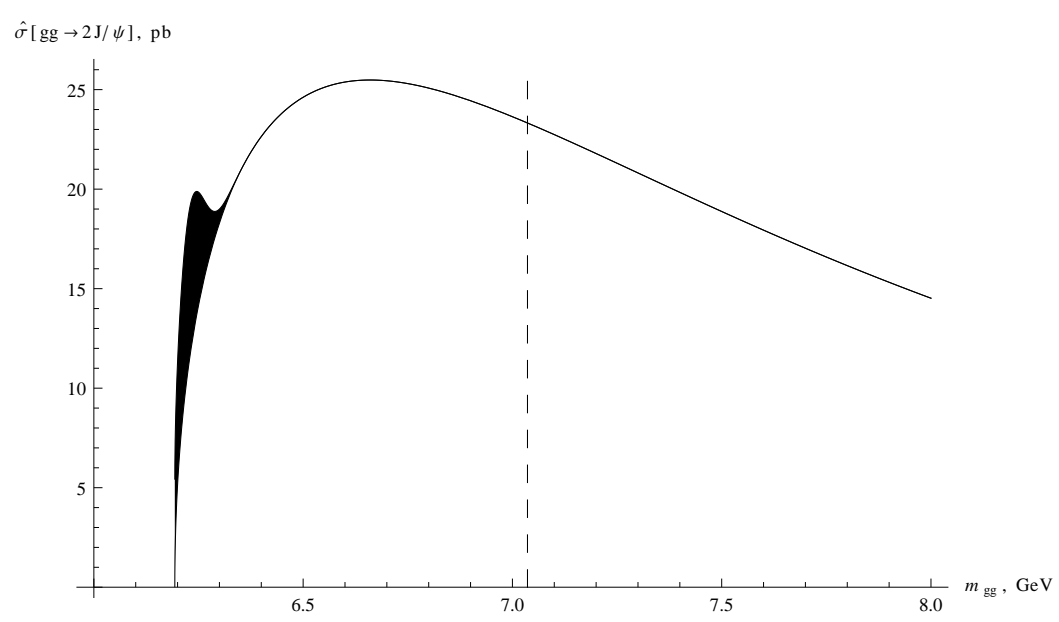

Figure 1: Invariant mass distribution of $J / \psi$-meson pairs with expected $T_{4 c}$ tetraquark contribution. Dashed vertical line corresponds to the two doubly-heavy baryons formation threshold $2 M_{\Xi_{c c}}$.

tetraquark would preferably decay into a vector quarkonia pair. Indeed, decay into light mesons is suppressed by the Zweig rule, production of 4 open heavy flavor mesons is prohibited kinematically and formation of pseudoscalar quarkonia requires flip of the heavy quark spin. That is why the following duality relation can be written:

$$
S_{T}=\int_{2 M_{\mathscr{Q}}}^{2 M_{\Xi_{Q Q}}} d m_{g g} \hat{\sigma}[g g \rightarrow T \rightarrow 2 \mathscr{Q}]=\varepsilon \int_{2 m_{[Q Q]}}^{2 M_{\Xi_{Q Q}}} d m_{g g} \hat{\sigma}\left(g g \rightarrow\left[Q_{1} Q_{2}\right]+\left[\bar{Q}_{3} \bar{Q}_{4}\right]\right),
$$

where $\varepsilon$ factor stands for the other possible decay modes. This value is to be compared with the integrated non-resonant cross section of quarkonia pairs production in the same duality window:

$$
S_{2 \mathscr{Q}}=\int_{2 M_{\mathscr{Q}}}^{2 M_{\Xi_{Q Q}}} d m_{g g} \hat{\sigma}[g g \rightarrow 2 \mathscr{Q}] .
$$

As tetraquark states are typically narrow, these mesons can be observed as peaks in the quarkonia pairs invariant mass distributions despite that $S_{T} \ll S_{2 \mathscr{Q}}$ relation holds. As tetraquark width is small compared to the detector resolution $\Delta \approx 50 \mathrm{MeV}$, its peak can be modeled by a Gaussian form with corresponding width. Therefore cross section of the $g g \rightarrow T \rightarrow 2 \mathscr{Q}$ Breit-Wigner process is replaced with the following expression:

$$
\hat{\sigma}(g g \rightarrow T \rightarrow 2 \mathscr{Q})=\frac{S_{T}}{\sqrt{\pi} \Delta} \exp \left\{-\frac{\left(m_{g g}-M_{T}\right)^{2}}{\Delta^{2}}\right\},
$$

where preexponential factor is selected according to the duality relation (3.1).

Let us begin with the tetraquarks composed of the 4 identical quarks. In the $T_{4 c}$ case only tensor state lies above the 2 vector charmonia production threshold. Integrated cross sections calculated using expressions (3.1) and (3.2) are equal

$$
S_{T_{4 c}}=0.7 \mathrm{pbGeV} \quad S_{2 J / \psi}=20 \mathrm{pb} \mathrm{GeV},
$$



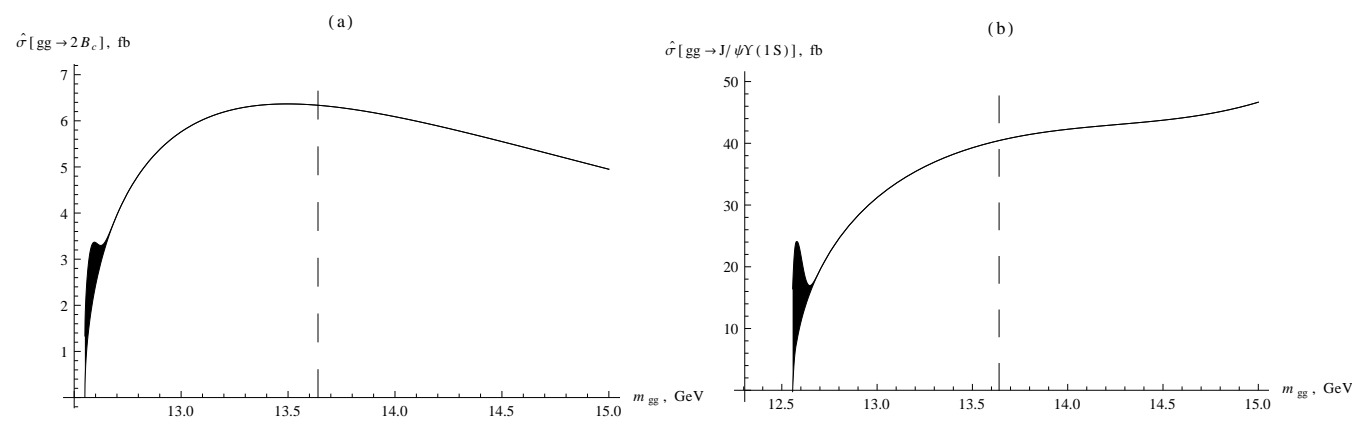

Figure 2: Invariant mass distribution of $B_{c}$-meson pairs (left plot) and $J / \psi \Upsilon(1 S)$ (right plot) with expected contribution of the $T_{2[b c]}$ tetraquark. Dashed vertical line corresponds to the two doubly-heavy baryons formation threshold $2 M_{\Xi_{b c}}$.

where suppression factor is selected to be $\varepsilon=0.2$. Invariant mass distribution for the $J / \psi$-meson pairs with expected $T_{4 c}$ tetraquark contribution is shown in Fig. 1.

As already mentioned, in the $T_{4 b}$ tetraquark case even tensor state is under the two vector bottomonia formation threshold so its observation in their invariant mass distribution is doubtful.

Let us turn to the $T_{2[b c]}=[b c][\bar{b} \bar{c}]$ tetraquark. In this case pseudoscalar $B_{c}$-meson and vector $B_{c^{-}}^{*}$ meson decaying into $B_{c} \gamma$ can be experimentally observed. Thus tensor tetraquark $T_{2[b c]}\left(2^{++}\right)$can be observed as a peak in the $B_{c}$-meson pairs invariant mass distribution. However $T_{2[b c]}\left(2^{++}\right) \rightarrow 2 B_{c}$ decay requires flip of the heavy quark spin and is suppressed by the factor

$$
\varepsilon \sim \frac{M_{T}-2 m_{[b c]}}{m_{[b c]}} \approx 2.6 \times 10^{-3} .
$$

Integrated cross sections (3.1) and (3.2) are equal

$$
S_{T_{2[b c]}}=0.13 \mathrm{fbGeV} \quad S_{2 B_{c}}=6 \mathrm{fbGeV},
$$

where $M_{\Xi_{b c}}=6.82 \mathrm{GeV}$ [19] is used. Invariant mass distribution of the $B_{c}$-meson pairs with expected contribution of the $T_{2[b c]}$ tetraquark is shown in Fig. 2a. All $T_{2[b c]}$ mesons lie under the $B_{c}^{*}$ pair production threshold.

Decay of the $T_{2[b c]}$ tetraquark into the $J / \psi \Upsilon(1 S)$ vector quarkonia pair is also possible. In the color singlet model reaction $g g \rightarrow J / \psi \Upsilon(1 S)$ is prohibited so accounting for octet components of vector quarkonia is needed. This process was elaborated in the work [20] results of which are used as the background for the tetraquark contribution. Suppression factor $\varepsilon \sim 3 \times 10^{-2}$ was used to obtain integrated cross sections (3.1) and (3.2):

$$
S_{T_{2[b c]}}=1.5 \mathrm{fbGeV}, \quad S_{\psi \Upsilon}=33 \mathrm{fb} \mathrm{GeV}
$$

Invariant mass distribution of the $J / \psi \Upsilon(1 S)$ pairs with expected contribution of the $T_{2[b c]}$ tetraquark is shown in Fig. $2 b$. 


\section{Conclusion}

In our work tetraquarks composed of 4 heavy quarks are concerned. Spectroscopy of $T_{4 c}=$ $[c c][\bar{c} \bar{c}], T_{4 b}=[b b][\bar{b} \bar{b}]$ and $T_{2 b c}=[b c][\bar{b} \bar{c}]$ states is elaborated and possibility of observation at the LHC is studied.

Model used implies diquark structure of tetraquarks i.e. $Q_{1} Q_{2} \bar{Q}_{3} \bar{Q}_{4}$ tetraquark is assumed to consist of 2 almost point-like diquarks $\left[Q_{1} Q_{2}\right]$ and $\left[\bar{Q}_{3} \bar{Q}_{4}\right]$ being in triplet color configuration. In such a treatment tetraquark is entirely analogous to a doubly-heavy meson. So non-relativistic Schroedinger equation which gives reliable results for the charmonia and bottomonia description can be used to obtain its parameters.

In the case of $T_{4 c}$ and $T_{4 b}$ tetraquarks Fermi principle imposes constraints on the possible quantum numbers of diquarks: in the antitriplet color configuration total spin of the $S$-wave diquark can be only equal to 1 . So with the hyperfine splitting accounted for 3 states with the following quantum numbers appear: $0^{++}, 1^{+-}$and $2^{++}$. In the charmed tetraquark case only tensor meson lies above the vector meson pair formation threshold and its peak can be observed at LHC in their invariant mass distribution. For the $T_{4 b}$ tetraquark all the states lie under the $\Upsilon(1 S)$ pair formation threshold. Both 0 and 1 spins are possible for the diquark in the $T_{2 b c}$ tetraquark. So 6 states arise after the hyperfine splitting of the unaffected state: two of $0^{++}$, one $1^{++}$, two of $1^{+-}$and one $2^{++}$.

In the last section we estimated the possibility to observe tetraquarks concerned in the inclusive reactions $g g \rightarrow T \rightarrow 2 \mathscr{Q}$. According to our calculations $T_{4 c}\left(2^{++}\right)$tensor state can be observed as a peak in the $J / \psi$-meson pairs invariant mass distribution. $T_{2[b c]}\left(2^{++}\right)$tetraquark can be observed in both $2 B_{c}$ and $J / \psi \Upsilon(1 S)$ modes.

\section{Acknowledgments}

Authors would like to thank A.K. Likhoded, V.V. Kiselev and A.A. Novoselov for the fruitful discussions. The work was financially supported by Russian Foundation for Basic Research (grant \#10-02-00061a), non-commercial foundation "Dynasty" and the grant of the president of Russian Federation (grants \#MK-406.2010.2, \#MK-3513.2012.2).

\section{References}

[1] R. L. Jaffe, Phys. Rev. D 15, 267 (1977).

[2] R. L. Jaffe, Phys. Rev. D 15, 281 (1977).

[3] L. Maiani, F. Piccinini, A. Polosa and V. Riquer, Phys. Rev. D 71, 014028 (2005).

[4] S. S. Gershtein, A. K. Likhoded and A. V. Luchinsky, Phys. Rev. D 74, 016002 (2006).

[5] N. Mathur et al., Phys. Rev. D 76, 114505 (2007).

[6] S. Prelovsek et al., PoS LAT2009, 103 (2009); arXiv:0910.2749.

[7] D0 Collab. (V. M. Abazov et al.), Phys. Rev. Lett. 93, 162002 (2004).

[8] BABAR Collab. (B. Aubert et al.), Phys. Rev. D 71, 071103 (2005).

[9] E. S. Swanson, Phys. Rept. 429, 243 (2006). 
[10] D. Ebert, R. N. Faustov and V. O. Galkin, Phys. Lett. B 634, 214 (2006).

[11] Belle Collab. (K. F. Chen et al.), Phys. Rev. Lett. 100, 112001 (2008).

[12] Belle Collab. (I. Adachi et al.), Phys. Rev. D 82, 091106(R) (2010).

[13] A. Ali, C. Hambrock and M. J. Aslam, Phys. Rev. Lett. 104, 162001 (2010).

[14] A. Ali, C. Hambrock and W. Wang, Tetraquark Interpretation of the Charged Bottomonium-like states $Z_{b}^{+,-}(10610)$ and $Z_{b}^{+,-}(10650)$ and Implications, arXiv:1110.1333.

[15] A. Berezhnoy, A. Likhoded, A. Luchinsky and A. Novoselov, Phys. Rev. D 84, 094023 (2011).

[16] K. Terasaki, Prog. Theor. Phys. 125, 199 (2011).

[17] V. Kiselev, A. Likhoded, O. Pakhomova and V. Saleev, Phys. Rev. D 66, 034030 (2002).

[18] A. Ali, C. Hambrock, I. Ahmed, and M. J. Aslam, Phys. Lett. B 684, 28 (2010).

[19] V. Kiselev and A. Likhoded, Phys. Usp. 45, 455 (2002).

[20] P. Ko, C. Yu, and J. Lee, JHEP 1101, 070 (2011). 